\title{
Effect of basis set superposition error on the electron density of molecular complexes
}

\author{
Pedro Salvador, Xavier Fradera, and Miquel Duran ${ }^{\mathrm{a})}$ \\ Institute of Computational Chemistry and Department of Chemistry, University of Girona, 17071 Girona, \\ Catalonia, Spain
}

(Received 3 March 2000; accepted 23 March 2000)

\begin{abstract}
The Chemical Hamiltonian Approach (CHA) versions of the Roothaan and Kohn-Sham equations, labeled CHA/F and CHA/DFT, respectively, have been used to obtain the basis set superposition error (BSSE)-corrected first-order electron density of the hydrogen fluoride dimer with several basis sets. We have analyzed the effect of BSSE in terms of the electronic relaxation, i.e., the redistribution of the electron density due to the inclusion of the CHA correction at a frozen geometry, along with the subsequent nuclear relaxation process. Critical points of the charge density have been located and characterized to compare the conventional, uncorrected first-order electron density against the BSSE-corrected density at each level of theory. Contour difference maps between BSSE-corrected and uncorrected densities on the molecular plane have also been plotted to gain insight into the effects of BSSE correction on the electron density. (C) 2000 American Institute of Physics. [S0021-9606(00)31423-4]
\end{abstract}

\section{INTRODUCTION}

Description of molecular complexes suffers from the socalled basis set superposition error (BSSE). ${ }^{1}$ This unphysical effect causes the overestimation of the interaction energy ${ }^{2}$ due to an imbalance between the description of the molecules that compose the molecular complex in the complex itself and their isolated description. For many years, the most common way to overcome this problem has been the socalled counterpoise (CP) method. ${ }^{3}$ This well-known procedure has been recently generalized and applied successfully to obtain BSSE-corrected geometries, harmonic frequencies, and any property that can be expressed as a derivative of the total energy of the molecular complexes. ${ }^{4}$ Unfortunately, the $\mathrm{CP}$ recipe does not furnish a corrected wave function (and thus a corrected electron density) from which one can obtain the CP-corrected energy as the expected value of the Hamiltonian operator of the system.

These shortcomings can be avoided by aprioristic methods like the Chemical Hamiltonian Approach (CHA), ${ }^{5-12}$ which are able to yield a corrected wave function and hence a BSSE-free electron density. CHA methods drop the terms causing pure BSSE delocalizations directly from the Hamiltonian, so the balance between the description of the fragments that compose the molecular complex in the complex itself and their isolated description is maintained. In the last decade, Mayer et al. have implemented the CHA methodology for the most common quantum mechanical ab initio methods. The corresponding CHA versions of Hartree-Fock theory $\left(\mathrm{CHA}-\mathrm{SCF},{ }^{5-7} \mathrm{CHA} / \mathrm{F}\right),{ }^{8}$ density functional theory (CHA/DFT) ${ }^{9}$ Mbller-Plesset perturbation theory CHA-MP2,${ }^{10}$ intermolecular perturbation theory PT-CHA, ${ }^{11}$ and configuration interaction $\mathrm{CHA}-\mathrm{CI}^{12}$ have been success-

\footnotetext{
a) Author to whom correspondence should be addressed; electronic mail: quel@iqc.udg.es
}

fully tested on hydrogen-bonded complexes and van der Waals systems. For instance, recent studies ${ }^{13,14}$ have shown that both the $\mathrm{CP}$ and the CHA approaches correct for BSSE in a very similar way for several hydrogen-bonded complexes at the SCF and DFT levels of theory, not only regarding interaction energies, but also concerning BSSE-corrected geometrical parameters.

The purpose of this paper is to proceed one step ahead on the analysis of the effect of BSSE on molecular complexes, and to assess how the artificial delocalizations caused by BSSE modify the first order electron density of a wellstudied system like the hydrogen fluoride dimer.

Numerical geometry optimizations of this system were carried out in a previous study ${ }^{13}$ with several basis sets using both the BSSE-free CHA versions of the Roothaan and DFT Kohn-Sham equations (labeled CHA/F and CHA/DFT, respectively), and the conventional Hartree-Fock (HF) and DFT methodologies. In the present paper, for each method we have computed the first-order electron density at the stationary points of the potential energy surface (PES) located with both the conventional method and its CHA counterpart.

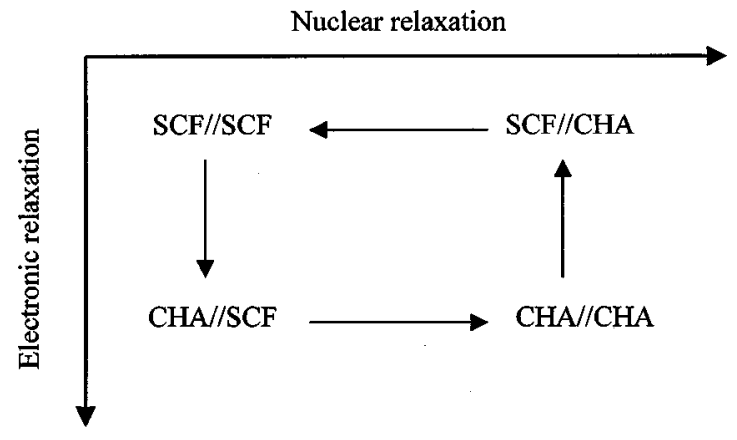

To gain insight into the changes between BSSEcorrected and-uncorrected densities, one can view the BSSE correction as a perturbation on the system, in analogy to the 
inclusion of electronic correlation with respect to a HF reference wave function. This approach for analyzing the effect of the BSSE correction on the electron density can be used indistinctly within the HF and DFT approximations. The situation is depicted in Scheme 1, where $X / / Y$ represents a single-point calculation using method $X$ at the geometry optimized with method $Y$. We will use this notation throughout the present paper. The analysis of the BSSE effect on the electron density will be carried out in the following way: the SCF//SCF point represents the unperturbed situation, where no BSSE correction has been taken into account. When the corresponding CHA method is applied over the SCF optimized geometry, the electron density is redistributed according to the new Hamiltonian introduced by the CHA. We denote this transition as $\mathrm{SCF} / / \mathrm{SCF} \rightarrow \mathrm{CHA} / \mathrm{SCF}$. The changes on the density associated to this transition can be assigned to the electronic relaxation induced by the BSSE correction. In a second step, the system is allowed to relax to its optimal geometry, induced by the new electrostatic potential. This process is represented by the $\mathrm{CHA} / \mathrm{SCF} \rightarrow \mathrm{CHA} / / \mathrm{CHA}$ transition, and accounts for nuclear relaxation effects. The elimination of the perturbation $(\mathrm{CHA} / / \mathrm{CHA} \rightarrow \mathrm{SCF} / / \mathrm{CHA})$ followed by a final nuclear relaxation (SCF//CHA $\rightarrow \mathrm{SCF} / \mathrm{SCF}$ ) would lead to the starting point. An analogous pathway holds in the case of the DFT level of theory. It is important to note that the CHA method is not a perturbation itself. However, by considering the BSSE correction as a perturbation one can study the BSSE correction in terms of two consecutive effects: the electronic redistribution and the nuclear relaxation.

The effect of the electronic relaxation can be easily visualized by means of contour maps of density differences between the conventional and the BSSE-corrected densities. For nuclear relaxation effects, electron densities computed at different nuclear arrangements can be compared through the atoms in molecules (AIM) theory ${ }^{15}$ by means of the characterization of the critical points on the electron density. Note that tackling the analysis of the BSSE correction is similar to the way that introduction of electronic correlation on a system is usually studied. Indeed, the analysis of the influence of the correlation energy on the bonding or the electron density is generally carried out considering also the electronic and nuclear relaxation effects separately. ${ }^{16}$

Recent studies ${ }^{17,18}$ have also dealt with the obtention of BSSE-corrected electron densities. The correction has been made by the SCF for molecular interactions ${ }^{19}$ (SCF-MI) approach, which restricts the expansion of the molecular orbitals of each fragment on its own atomic orbitals, thus leading to a block-diagonal density matrix. Because Mulliken charge transfer between the fragments is then zero by definition, this method also eliminates some true interaction terms, overestimating the BSSE not only with respect to CHA but also the CP method. A second main drawback of the SCF-MI method is that as opposed again to both the CP and CHA approaches, it does not exhibit the desired asymptotic behavior with the increase of basis set size. ${ }^{17}$

This paper deals with densities obtained by means of the CHA approach, which overcomes the problems shown by other BSSE-corrected methods. This study is organized in the following way: in Sec. II we will shortly comment on the main features of the BSSE-corrected methods used in the present paper: in Sec. III we will describe the computational procedure, whereas in Sec. IV we will analyze the results obtained in terms of the electronic and nuclear relaxation effects caused by the BSSE.

\section{CHA/F AND CHA/DFT METHODS}

A wide number of publications on the topic and a recent review ${ }^{20}$ are available, so we shall not go into detail on the CHA methodology, and we will describe just its main features. The main point of the CHA philosophy is to detect and remove from the Hamiltonian those intermolecular contributions that cause the BSSE, while keeping all true interaction terms, so that the description of each atom (fragment) using all the basis functions of the molecule is consistent with the description of the isolated atom (fragment). For this purpose, a mixed second-quantization formalism is used to expand the Hamiltonian into the atomic orbital basis and to separate the intramolecular, the pure intermolecular, and the BSSE-pure contributions. After these modifications are performed, the resulting Hamiltonian is no longer hermitian, so application of the variational principle (under the monodeterminantal approximation) to derive the SCF equations is not trivial. However, by making use of the Brillouin theorem ${ }^{5}$ it is still possible to derive a set of self-consistent equations analogous to the conventional HF ones to obtain the so-called CHA-SCF canonic orbitals.

In the so-called $\mathrm{CHA} / \mathrm{F}$ method, any modifications are performed directly to the standard Fock equations, and hence the one- and two-electron integrals remain unchanged. The changes on the Roothaan equations are minor and the extra computational cost is negligible when compared to the twoelectron integral handling effort. Even though the CHA/F equations can be seen as an approximation to the CHA-SCF ones, where fulfillment of the Brillouin condition is implicitly ensured, experience shows that $\mathrm{CHA} / \mathrm{F}$ and $\mathrm{CHA}-\mathrm{SCF}$ results are almost equivalent.

As the Hamiltonian is non-hermitian, its eigenfunctions are not orthonormalized and they can even appear as pairs of complex conjugated roots at each step of the SCF procedure. It would be a difficult task to obtain the first-order density matrix from a wave function made up of nonorthogonal orbitals, so in order to build up the density matrix for the next iteration, the occupied orbitals are orthonormalized among themselves. It is well known from their properties that the Slater determinants built from the nonorthogonal and the orthogonal molecular orbital sets differ only by an unimportant constant factor. Therefore, when self-consistency is achieved, the canonic CHA orbitals obtained are orthonormalized so that the first-order density matrix can be obtained in the conventional way.

The fact that the energy must be computed with the original Hamiltonian, together with the nonorthogonal nature of the resulting molecular orbitals, has as a direct consequence that the evaluation of the analytic gradient is not at all straightforward. Although the corresponding equations have already been derived, ${ }^{21}$ they have not been implemented yet, so one is forced to use numerical gradients ob- 

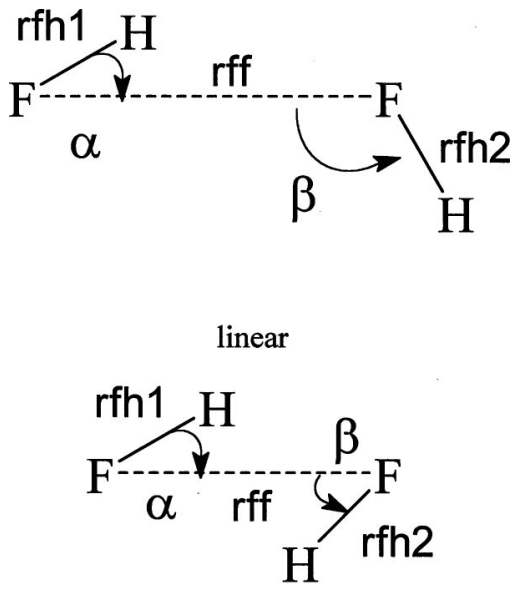

\section{box}

FIG. 1. Geometrical parameters of the hydrogen fluoride dimer for the linear and the cyclic structures.

tained by finite differences in order to locate the stationary points on the BSSE-corrected PES.

\section{COMPUTATIONAL DETAILS}

First-order electron densities were calculated using the GAUSSIAN $94^{22}$ package at the HF and DFT levels of theory using the $6-31 \mathrm{G}, 6-31 \mathrm{G}(\mathrm{d}), 6-31 \mathrm{G}(\mathrm{d}, \mathrm{p}), 6-31++\mathrm{G}(\mathrm{d}, \mathrm{p})$, $6-311 \mathrm{G}(\mathrm{d}, \mathrm{p})$, and $6-311++\mathrm{G}(3 \mathrm{df}, 2 \mathrm{pd})$ basis sets at the optimized structures ${ }^{13}$ reported earlier. In DFT calculations, the nonlocal BLYP and the hybrid B3LYP functionals were used. In some cases, we performed new geometry optimizations having a tighter convergence criterion $\left(10^{-6}\right.$ in the rms of the gradient). Recalculated intermolecular angles differed by less than $1^{\circ}$ in all cases. For the CHA/F and CHA/DFT calculations we used a modified version of GAUSSIAN92. ${ }^{23}$
The new stationary points were located on the BSSEcorrected PES using numerical gradients and a tight convergence criterion.

The critical points of the charge density were located and characterized by means of the AIMPAC ${ }^{24}$ package. Selected parameters like the values of charge density and its Laplacian at the critical points of the electron density were used to determine quantitatively the differences between the uncorrected and CHA-corrected densities.

\section{RESULTS AND DISCUSSION}

The geometrical parameters and electronic interaction energies of the hydrogen fluoride dimer (see Fig. 1) for selected basis sets at the SCF, BLYP, and B3LYP levels of theory together with their respective CHA counterparts are collected in Tables I-III respectively. We will comment here on just the general trends concerning the effect of BSSE on the geometry in order to better understand the effect of nuclear relaxation on the electron density. For a deeper discussion see Ref. 13.

It can be seen that intermolecular distances optimized on the CHA PES $(r \mathrm{~F}-\mathrm{F})$ are always longer that the corresponding BSSE-uncorrected ones, whereas the intramolecular parameters (rfh1 and rfh2) remain almost unchanged. The lengthening of the intermolecular distance leads to a decrease in the interaction energy, after correction for BSSE. Tables I-III also show the importance of using diffuse functions for the correct description of the systems and the minimization of BSSE. However, the use of the CHA methodology seems to ensure correct descriptions ${ }^{25}$ of the systems even with small basis sets. A better agreement with the experimental parameters is obtained in all cases, but for the $\mathrm{SCF} / 6-31 \mathrm{G}$ calculation. Finally, as expected, the uncorrected and the CHA molecular parameters (energies, atomic distances) tend to each other as the size of the basis set increases, converging to the same value in the complete basis set limit. All these

TABLE I. Geometrical parameters ( $\AA$ and deg) and electronic interaction energy $(\mathrm{kcal} / \mathrm{mol})$ for the HF-HF dimer calculated in ten different basis sets at the SCF and CHA/F corrected levels of theory. The experimental ${ }^{\mathrm{a}}$ values of $r \mathrm{~F}-\mathrm{F}, \alpha$, and $\beta$ are $2.72 \AA, 10^{\circ} \pm 6^{\circ}$, and $117^{\circ} \pm 10^{\circ}$, respectively. The number of basis functions is shown in parenthesis.

\begin{tabular}{|c|c|c|c|c|c|c|c|}
\hline Basis set & Method & $r f h 1$ & $r \mathrm{~F}-\mathrm{F}$ & $r f h 2$ & $\alpha$ & $\beta$ & $\begin{array}{c}\text { Interaction } \\
\text { energy }\end{array}$ \\
\hline $6-31 \mathrm{G}$ & SCF & 0.925 & 2.706 & 0.923 & 8.3 & 126.0 & -7.49 \\
\hline$(22)$ & $\mathrm{CHA} / \mathrm{F}$ & 0.925 & 2.713 & 0.923 & 5.1 & 135.6 & -6.55 \\
\hline $6-31 G(d)$ & SCF & 0.915 & 2.709 & 0.915 & 17.1 & 96.6 & -6.06 \\
\hline (34) & $\mathrm{CHA} / \mathrm{F}$ & 0.915 & 2.756 & 0.914 & 7.6 & 114.8 & -4.73 \\
\hline $6-31 G(d, p)$ & SCF & 0.905 & 2.725 & 0.904 & 14.4 & 101.7 & -5.97 \\
\hline$(40)$ & $\mathrm{CHA} / \mathrm{F}$ & 0.904 & 2.760 & 0.903 & 8.3 & 116.7 & -4.62 \\
\hline $6-31++\mathrm{G}(\mathrm{d}, \mathrm{p})$ & SCF & 0.906 & 2.812 & 0.905 & 8.0 & 120.0 & -4.37 \\
\hline$(50)$ & $\mathrm{CHA} / \mathrm{F}$ & 0.906 & 2.831 & 0.905 & 7.4 & 120.8 & -4.14 \\
\hline $6-311 \mathrm{G}(\mathrm{d}, \mathrm{p})$ & $\mathrm{SCF}$ & 0.900 & 2.773 & 0.899 & 11.6 & 112.6 & -5.20 \\
\hline$(50)$ & $\mathrm{CHA} / \mathrm{F}$ & 0.900 & 2.822 & 0.899 & 7.7 & 122.8 & -4.45 \\
\hline $6-311++\mathrm{G}(3 \mathrm{df}, 2 \mathrm{pd})$ & SCF & 0.902 & 2.821 & 0.900 & 7.0 & 118.7 & -3.93 \\
\hline$(122)$ & $\mathrm{CHA} / \mathrm{F}$ & 0.901 & 2.840 & 0.900 & 7.2 & 119.9 & -3.69 \\
\hline
\end{tabular}

${ }^{\mathrm{a}}$ See Ref. 27. 
TABLE II. Geometrical parameters $(\AA$ and deg) and electronic interaction energy ( $\mathrm{kcal} / \mathrm{mol})$ for the $\mathrm{HF}-\mathrm{HF}$ dimer calculated in ten different basis sets at the BLYP and CHA/DFT(BLYP) corrected levels of theory. The experimental $^{\mathrm{a}}$ values of $r \mathrm{~F}-\mathrm{F}, \alpha$, and $\beta$ are $2.72 \AA, 10^{\circ} \pm 6^{\circ}$, and $117^{\circ} \pm 10^{\circ}$, respectively. The number of basis functions is shown in parenthesis.

\begin{tabular}{|c|c|c|c|c|c|c|c|}
\hline Basis set & Method & $r f h 1$ & $r \mathrm{~F}-\mathrm{F}$ & $r f h 2$ & $\alpha$ & $\beta$ & $\begin{array}{c}\text { Interaction } \\
\text { energy }\end{array}$ \\
\hline $6-31 \mathrm{G}$ & BLYP & 0.972 & 2.480 & 0.972 & 46.9 & 47.0 & -11.16 \\
\hline$(22)$ & CHA/BLYP & 0.972 & 2.662 & 0.964 & 5.6 & 114.9 & -7.27 \\
\hline $6-31 G(d)$ & BLYP & 0.954 & 2.485 & 0.954 & 45.0 & 45.0 & -10.52 \\
\hline (34) & CHA/BLYP & 0.954 & 2.652 & 0.949 & 5.2 & 105.9 & -5.88 \\
\hline $6-31 G(d, p)$ & BLYP & 0.946 & 2.494 & 0.946 & 44.8 & 45.0 & -10.45 \\
\hline$(40)$ & CHA/BLYP & 0.946 & 2.626 & 0.940 & 7.6 & 105.2 & -5.71 \\
\hline $6-31++\mathrm{G}(\mathrm{d}, \mathrm{p})$ & BLYP & 0.947 & 2.760 & 0.942 & 7.5 & 111.6 & -4.75 \\
\hline$(50)$ & CHA/BLYP & 0.947 & 2.782 & 0.942 & 7.4 & 110.6 & -4.45 \\
\hline $6-311 \mathrm{G}(\mathrm{d}, \mathrm{p})$ & BLYP & 0.938 & 2.572 & 0.938 & 46.8 & 48.6 & -8.28 \\
\hline$(50)$ & CHA/BLYP & 0.939 & 2.730 & 0.934 & 8.8 & 107.9 & -5.41 \\
\hline $6-311++\mathrm{G}(3 \mathrm{df}, 2 \mathrm{pd})$ & BLYP & 0.941 & 2.768 & 0.937 & 5.3 & 112.0 & -4.40 \\
\hline$(122)$ & CHA/BLYP & 0.941 & 2.778 & 0.936 & 4.8 & 113.0 & -4.16 \\
\hline
\end{tabular}

${ }^{a}$ See Ref. 27.

general trends have been observed for all systems studied so far, including both hydrogen bonds ${ }^{13}$ and Van der Waals interactions. ${ }^{26}$

As mentioned in Ref. 13, an interesting situation arises in the case of the hydrogen fluoride dimer. The results obtained for the angular parameters (see Tables I-III) indicate that optimizations using the $6-31 \mathrm{G}(\mathrm{d}), 6-31 \mathrm{G}(\mathrm{d}, \mathrm{p})$, and even 6-31G $(\mathrm{d}, \mathrm{p})$ basis sets lead to linear distorted structures for the SCF calculations, and to cyclic structures when using both the BLYP and the B3LYP functionals. Note that the corresponding CHA calculations change this situation and lead to the correct structures and interaction energies as compared to the experiment. ${ }^{27}$ These kinds of dramatic changes in the topology of the PES upon the BSSE correction have also been observed in other weakly bound systems like the water dimer $^{13}$ or some $\mathrm{C}-\mathrm{H} \cdots \mathrm{O}$ hydrogen bonded complexes. ${ }^{28}$ Both $\mathrm{CHA}$ and $\mathrm{CP}$ results point out that this effect is directly caused by the BSSE rather than by the supposed poor behavior of the actual functionals used to describe these kinds of systems. ${ }^{29}$

By studying the PES one can extract information mainly in terms of nuclear relaxation, i.e., how the nuclear positions move according to the electronic potential. To gain a deeper insight into how the BSSE affects the electronic distribution at a given nuclear arrangement (electronic relaxation) one is driven to study the electron density and its topology. In order to compare both the uncorrected and the CHA first order electron densities, we will use the well-know AIM theory,

TABLE III. Geometrical parameters ( $\AA$ and deg) and electronic interaction energy (kcal/mol) for the HF-HF dimer calculated in ten different basis sets at the B3LYP and CHA/DFT(B3LYP) corrected levels of theory. The experimental $^{\mathrm{a}}$ values of $r \mathrm{~F}-\mathrm{F}, \alpha$ and $\beta$ are $2.72 \AA, 10^{\circ} \pm 6^{\circ}$, and $117^{\circ} \pm 10^{\circ}$, respectively. The number of basis functions is shown in parenthesis.

\begin{tabular}{|c|c|c|c|c|c|c|c|}
\hline Basis set & Method & $r f h 1$ & $r \mathrm{~F}-\mathrm{F}$ & $r f h 2$ & $\alpha$ & $\beta$ & $\begin{array}{c}\text { Interaction } \\
\text { energy }\end{array}$ \\
\hline $6-31 G$ & B3LYP & 0.956 & 2.478 & 0.956 & 48.7 & 49.6 & -10.20 \\
\hline$(22)$ & CHA/B3LYP & 0.957 & 2.647 & 0.951 & 5.2 & 120.0 & -7.56 \\
\hline $6-31 G(d)$ & B3LYP & 0.941 & 2.485 & 0.941 & 46.4 & 46.6 & -9.60 \\
\hline (34) & CHA/B3LYP & 0.942 & 2.647 & 0.938 & 5.7 & 107.5 & -6.15 \\
\hline $6-31 G(d, p)$ & B3LYP & 0.933 & 2.493 & 0.933 & 46.4 & 46.5 & -9.42 \\
\hline (40) & CHA/B3LYP & 0.933 & 2.627 & 0.929 & 7.8 & 107.5 & -5.87 \\
\hline $6-31++\mathrm{G}(\mathrm{d}, \mathrm{p})$ & B3LYP & 0.935 & 2.732 & 0.931 & 7.6 & 113.1 & -5.08 \\
\hline$(50)$ & CHA/B3LYP & 0.935 & 2.752 & 0.931 & 7.4 & 112.5 & -4.79 \\
\hline $6-311 G(d, p)$ & B3LYP & 0.926 & 2.567 & 0.926 & 47.9 & 50.5 & -7.53 \\
\hline$(50)$ & CHA/B3LYP & 0.927 & 2.725 & 0.924 & 7.0 & 113.9 & -5.52 \\
\hline $6-311++\mathrm{G}(3 \mathrm{df}, 2 \mathrm{pd})$ & B3LYP & 0.930 & 2.734 & 0.926 & 5.8 & 112.5 & -4.71 \\
\hline$(122)$ & CHA/B3LYP & 0.930 & 2.747 & 0.926 & 5.4 & 113.9 & -4.47 \\
\hline
\end{tabular}

${ }^{a}$ See Ref. 27. 
TABLE IV. Electron density and its Laplacian at the intermolecular bond critical points located on the SCF and CHA/F electron densities calculated with several basis sets. The distance of the bcp to the fluorine atom of the $\mathrm{H}$-acceptor molecule is also reported.

\begin{tabular}{|c|c|c|c|c|}
\hline Basis set & $\begin{array}{l}\text { Method// } \\
\text { Geometry }\end{array}$ & $\begin{array}{c}\rho(r) \\
\left(e / \text { a.u. }{ }^{3}\right)\end{array}$ & $\begin{array}{l}\nabla^{2} \rho(r) \\
\left(\text { e/a.u. }{ }^{5}\right)\end{array}$ & $\begin{array}{c}\text { Distance to F } \\
(\AA)\end{array}$ \\
\hline \multirow{3}{*}{$6-31 \mathrm{G}$} & $\mathrm{SCF} / \mathrm{SCF}$ & 0.0285 & 0.1242 & 1.153 \\
\hline & $\mathrm{CHA} / \mathrm{SCF}$ & 0.0278 & 0.1302 & 1.150 \\
\hline & $\mathrm{CHA} / / \mathrm{CHA}$ & 0.0272 & 0.1304 & 1.147 \\
\hline \multirow{3}{*}{$6-31 G(d)$} & $\mathrm{SCF} / \mathrm{SCF}$ & 0.0252 & 0.1041 & 1.195 \\
\hline & $\mathrm{CHA} / / \mathrm{SCF}$ & 0.0241 & 0.1114 & 1.192 \\
\hline & $\mathrm{CHA} / / \mathrm{CHA}$ & 0.0231 & 0.1065 & 1.193 \\
\hline \multirow{3}{*}{$6-31 G(d, p)$} & $\mathrm{SCF} / \mathrm{SCF}$ & 0.0222 & 0.0946 & 1.228 \\
\hline & $\mathrm{CHA} / \mathrm{SCF}$ & 0.0213 & 0.0961 & 1.223 \\
\hline & $\mathrm{CHA} / / \mathrm{CHA}$ & 0.0199 & 0.0914 & 1.231 \\
\hline \multirow{3}{*}{$6-31 \mathrm{G}++(\mathrm{d}, \mathrm{p})$} & $\mathrm{SCF} / \mathrm{SCF}$ & 0.0175 & 0.0763 & 1.262 \\
\hline & $\mathrm{CHA} / \mathrm{SCF}$ & 0.0177 & 0.0750 & 1.263 \\
\hline & $\mathrm{CHA} / / \mathrm{CHA}$ & 0.0169 & 0.0713 & 1.273 \\
\hline \multirow{3}{*}{$6-311 \mathrm{G}(\mathrm{d}, \mathrm{p})$} & $\mathrm{SCF} / \mathrm{SCF}$ & 0.0227 & 0.1189 & 1.201 \\
\hline & $\mathrm{CHA} / \mathrm{SCF}$ & 0.0235 & 0.1129 & 1.201 \\
\hline & $\mathrm{CHA} / / \mathrm{CHA}$ & 0.0167 & 0.0875 & 1.258 \\
\hline \multirow{3}{*}{$6-311 \mathrm{G}++(3 \mathrm{df}, 2 \mathrm{pd})$} & $\mathrm{SCF} / \mathrm{SCF}$ & 0.0173 & 0.0779 & 1.246 \\
\hline & $\mathrm{CHA} / \mathrm{SCF}$ & 0.0180 & 0.0725 & 1.246 \\
\hline & $\mathrm{CHA} / / \mathrm{CHA}$ & 0.0170 & 0.0697 & 1.258 \\
\hline
\end{tabular}

characterizing the topology of the electronic distributions in terms of the nature of its critical points and values of the electron density and its Laplacian. Note that since no BSSEcorrected densities can be obtained by means of the CP method, this analysis can be carried out only by using $a$ priori methods for elimination of the BSSE.

Table IV-VI summarize the results obtained at the uncorrected SCF, BLYP, and B3LYP levels of theory and their CHA counterparts, respectively. For each basis set and level of theory, three calculations of the electron density have been performed as a result of the combination of two different methods (uncorrected and CHA) and its respective optimized geometries. Different conclusions can be drawn depending upon the use of DFT, which accounts for inclusion of dynamic electron correlation. Therefore we will discuss the SCF and DFT results separately. At each level of theory, we will concentrate first on the analysis of the electron density redistribution caused by the BSSE correction by means of density difference maps and the changes on the charge density and its Laplacian at the critical points. Finally we will assess the effect of the later nuclear relaxation by comparing again the features of the bond critical points (bcps) located on the corresponding density.

TABLE V. Electron density and its Laplacian at the intermolecular critical point located on the BLYP and CHA/BLYP first order electron densities calculated with several basis sets. The distance of the corresponding critical point to the fluorine atom of the $\mathrm{H}$-acceptor molecule is also reported.

\begin{tabular}{|c|c|c|c|c|c|}
\hline Basis set & $\begin{array}{l}\text { Method// } \\
\text { Geometry }\end{array}$ & $\begin{array}{c}\text { Critical } \\
\text { point }\end{array}$ & $\begin{array}{c}\rho(r) \\
\left(\text { e/a.u. }{ }^{3}\right)\end{array}$ & $\begin{array}{c}\nabla^{2} \rho(r) \\
\left(\text { e/a.u. }{ }^{5}\right)\end{array}$ & $\begin{array}{c}\text { Distance to } F \\
(\AA)\end{array}$ \\
\hline \multirow{5}{*}{$6-31 \mathrm{G}$} & BLYP//BLYP ${ }^{\mathrm{a}}$ & $(3,-1)^{\mathrm{b}}$ & 0.0260 & 0.1111 & $\cdots$ \\
\hline & & $(3,+1)$ & 0.0250 & 0.1262 & 1.245 \\
\hline & $\mathrm{CHA} / \mathrm{BLYP}^{\mathrm{a}}$ & $(3,-1)^{\mathrm{b}}$ & 0.0245 & 0.1218 & $\ldots$ \\
\hline & & $(3,+1)$ & 0.0240 & 0.1289 & 1.249 \\
\hline & $\mathrm{CHA} / / \mathrm{CHA}$ & $(3,-1)$ & 0.0398 & 0.1555 & 1.095 \\
\hline \multirow{5}{*}{$6-31 G(d)$} & BLYP//BLYP ${ }^{\mathrm{a}}$ & $(3,-1)^{\mathrm{b}}$ & 0.0270 & 0.1283 & $\ldots$ \\
\hline & & $(3,+1)$ & 0.0268 & 0.1403 & 1.246 \\
\hline & $\mathrm{CHA} / \mathrm{BLYP}^{\mathrm{a}}$ & $(3,-1)^{\mathrm{b}}$ & 0.0257 & 0.1399 & $\cdots$ \\
\hline & & $(3,+1)$ & 0.0256 & 0.1449 & 1.252 \\
\hline & $\mathrm{CHA} / / \mathrm{CHA}$ & $(3,-1)$ & 0.0384 & 0.1406 & 1.108 \\
\hline \multirow{4}{*}{$6-31 G(d, p)$} & BLYP//BLYP & $(3,-1)^{\mathrm{b}}$ & 0.0271 & 0.1345 & $\cdots$ \\
\hline & & $(3,+1)$ & 0.0271 & 0.1402 & 1.254 \\
\hline & $\mathrm{CHA} / \mathrm{BLYP}^{\mathrm{a}}$ & $(3,-1)$ & 0.0259 & 0.1443 & 1.238 \\
\hline & $\mathrm{CHA} / / \mathrm{CHA}$ & $(3,-1)$ & 0.0380 & 0.1277 & 1.120 \\
\hline \multirow{3}{*}{$6-31 \mathrm{G}++(\mathrm{d}, \mathrm{p})$} & BLYP//BLYP & $(3,-1)$ & 0.0264 & 0.0845 & 1.192 \\
\hline & CHA//BLYP & $(3,-1)$ & 0.0267 & 0.0807 & 1.191 \\
\hline & $\mathrm{CHA} / / \mathrm{CHA}$ & $(3,-1)$ & 0.0254 & 0.0760 & 1.203 \\
\hline \multirow{3}{*}{$6-311 \mathrm{G}(\mathrm{d}, \mathrm{p})$} & BLYP//BLYP ${ }^{\mathrm{a}}$ & $(3,-1)$ & 0.0221 & 0.0968 & 1.260 \\
\hline & $\mathrm{CHA} / \mathrm{BLYP}^{\mathrm{a}}$ & $(3,-1)$ & 0.0214 & 0.0996 & 1.264 \\
\hline & $\mathrm{CHA} / / \mathrm{CHA}$ & $(3,-1)$ & 0.0286 & 0.1077 & 1.179 \\
\hline \multirow{3}{*}{$6-311 \mathrm{G}++(3 \mathrm{df}, 2 \mathrm{pd})$} & BLYP//BLYP & $(3,-1)$ & 0.0263 & 0.0848 & 1.185 \\
\hline & CHA//BLYP & $(3,-1)$ & 0.0271 & 0.0789 & 1.186 \\
\hline & $\mathrm{CHA} / / \mathrm{CHA}$ & $(3,-1)$ & 0.0265 & 0.0775 & 1.191 \\
\hline
\end{tabular}

${ }^{\mathrm{a}}$ Cyclic structures.

bonly the features of one of the bcp's are reported, as both are almost equivalent by symmetry. 
TABLE VI. Electron density and its Laplacian at the intermolecular critical point located on the B3LYP and CHA/B3LYP first order electron densities calculated with several basis sets. The distance of the corresponding critical point to the fluorine atom of the $\mathrm{H}$-acceptor molecule is also presented.

\begin{tabular}{|c|c|c|c|c|c|}
\hline Basis set & $\begin{array}{l}\text { Method// } \\
\text { Geometry }\end{array}$ & $\begin{array}{l}\text { Critical } \\
\text { point }\end{array}$ & $\begin{array}{c}\rho(r) \\
\left(e / \text { a.u. }{ }^{3}\right)\end{array}$ & $\begin{array}{l}\nabla^{2} \rho(r) \\
\left(\text { e/a.u. }{ }^{5}\right)\end{array}$ & $\begin{array}{c}\text { Distance to } \mathrm{F} \\
(\AA)\end{array}$ \\
\hline \multirow{4}{*}{$6-31 \mathrm{G}$} & B3LYP//B3LYP & $(3,-1)^{\mathrm{b}}$ & 0.0240 & 0.1106 & $\cdots$ \\
\hline & & $(3,+1)$ & 0.0233 & 0.1207 & 1.259 \\
\hline & $\mathrm{CHA} / / \mathrm{B} 3 \mathrm{LYP} \mathrm{P}^{\mathrm{a}}$ & $(3,-1)$ & 0.0229 & 0.1186 & 1.199 \\
\hline & $\mathrm{CHA} / / \mathrm{CHA}$ & $(3,-1)$ & 0.0387 & 0.1588 & 1.096 \\
\hline \multirow{3}{*}{$6-31 G(d)$} & B3LYP//B3LYP & $(3,-1)$ & 0.0256 & 0.1287 & 1.207 \\
\hline & CHA//B3LYP & $(3,-1)$ & 0.0244 & 0.1380 & 1.210 \\
\hline & $\mathrm{CHA} / / \mathrm{CHA}$ & $(3,-1)$ & 0.0366 & 0.1409 & 1.114 \\
\hline \multirow{3}{*}{ 6-31G(d,p) } & B3LYP//B3LYPa & $(3,-1)$ & 0.0254 & 0.1312 & 1.212 \\
\hline & $\mathrm{CHA} / / \mathrm{B} 3 \mathrm{LYP} \mathrm{P}^{\mathrm{a}}$ & $(3,-1)$ & 0.0242 & 0.1399 & 1.222 \\
\hline & $\mathrm{CHA} / / \mathrm{CHA}$ & $(3,-1)$ & 0.0353 & 0.1278 & 1.131 \\
\hline \multirow{3}{*}{$6-31 \mathrm{G}++(\mathrm{d}, \mathrm{p})$} & B3LYP//B3LYP & $(3,-1)$ & 0.0263 & 0.0929 & 1.189 \\
\hline & CHA//B3LYP & $(3,-1)$ & 0.0267 & 0.0885 & 1.188 \\
\hline & $\mathrm{CHA} / / \mathrm{CHA}$ & $(3,-1)$ & 0.0254 & 0.0837 & 1.199 \\
\hline \multirow{3}{*}{ 6-311G(d,p) } & B3LYP//B3LYP & $(3,-1)$ & 0.0210 & 0.0950 & 1.246 \\
\hline & $\mathrm{CHA} / \mathrm{B} 3 \mathrm{LYP} \mathrm{P}^{\mathrm{a}}$ & $(3,-1)$ & 0.0204 & 0.0978 & 1.248 \\
\hline & $\mathrm{CHA} / / \mathrm{CHA}$ & $(3,-1)$ & 0.0272 & 0.1103 & 1.183 \\
\hline \multirow{3}{*}{$6-311 \mathrm{G}++(3 \mathrm{df}, 2 \mathrm{pd})$} & B3LYP//B3LYP & $(3,-1)$ & 0.0268 & 0.0905 & 1.179 \\
\hline & CHA//B3LYP & $(3,-1)$ & 0.0277 & 0.0849 & 1.180 \\
\hline & $\mathrm{CHA} / / \mathrm{CHA}$ & $(3,-1)$ & 0.0267 & 0.0827 & 1.186 \\
\hline
\end{tabular}

${ }^{\mathrm{a} C y c l i c}$ structures.

bonly the features of one of the bcp's are reported, as both are almost equivalent by symmetry.

\section{A. SCF}

The results for the topological analysis of SCF electron densities are collected in Table IV. In all cases a bcp between the hydrogen donor (H-donor) and the hydrogen acceptor ( $\mathrm{H}$-acceptor) molecules is located on the electron density. The values of the electron density and its Laplacian at this point are typical of a closed-shell weak interaction. The large distance of the bcp to the $\mathrm{F}$ atom of the $\mathrm{H}$-acceptor molecule also reveals this situation. Deviations caused by the BSSE are small in magnitude and, in general, represent about 5\%$10 \%$ of the value of $\rho(r)$ and $\nabla^{2} \rho(r)$ in the bcp. Note that the improvement of the quality of the basis set seems not to be the main factor as, for instance, the differences represent up to $25 \%$ for the large triple-zeta $6-311 \mathrm{G}(\mathrm{d}, \mathrm{p})$ calculation. As expected both the $6-31 \mathrm{G}++(\mathrm{d}, \mathrm{p})$ and the $6-311 \mathrm{G}++(3 \mathrm{df}, 2 \mathrm{p})$, the largest basis set used for this study, present the smallest differences, although these are still appreciable for the Laplacian, which is known to be a parameter very sensitive to small perturbations in the electron density.

Concerning the electronic relaxation, the following trends have been observed. For the small basis sets, the $\mathrm{SCF} / \mathrm{SCF} \rightarrow \mathrm{CHA} / \mathrm{SCF}$ transition causes a slight displacement of the bcp toward the $\mathrm{F}$ atom of the $\mathrm{H}$-acceptor molecule, a subsequent reduction of the value of the electron density and an increase of charge depletion at the bcp. However, when using larger basis sets including diffuse functions, or even with the 6-311G(d,p) basis set, the opposite effect is found. To better understand these effects, Fig. 2 depicts the density difference maps obtained at the uncorrected geometry for several basis sets. Solid lines represent the zones where the electron density is overestimated by BSSE. The maps obtained with basis sets without diffuse functions are qualitatively very similar [see Figs. 2(a), 2(b), and 2(c)]. The main differences between the conventional and the CHA densities are found in the atomic basins of the $\mathrm{F}$ atoms, mostly for the $\mathrm{H}$-acceptor moiety. It can be observed that the elimination of the BSSE transfers electron density from the intramolecular $\mathrm{F}-\mathrm{H}$ bonds to the $\mathrm{F}$ lone electron pairs. The electron density on the $\mathrm{H}$ atom regions is also increased, allowing for a minor charge transfer, and hence decreasing the intermolecular bond strength. Finally, the electron density in the intermolecular bond region is slightly overestimated by BSSE, except for the $6-311 \mathrm{G}(\mathrm{d}, \mathrm{p})$ basis set, for which this positive region in the map is not observed [see Fig. 2(e)]. This overestimation is connected with the increasing value of $\rho(r)$ at the bcp after electronic relaxation, as commented on above.

Adding diffuse functions changes the situation dramatically, because differences between the corrected and uncorrected densities become less important. Isocontour lines are more separated in the vicinity of the F atoms [see Figs. 2(d) and 2(f)]. An interesting point is that the BSSE effect tends to underestimate the electron density in the intermolecular bond region. This situation, opposite to that observed for smaller basis sets, can be explained in the following way: for any basis set (except for the complete basis set limit), the atomic orbitals of the hydrogen involved in the intermolecular bond can be used by the $\mathrm{F}$ atom of the $\mathrm{H}$-acceptor molecule to become more stable, causing the BSSE-like delocalizations. The CHA correction tends to increase the electron 


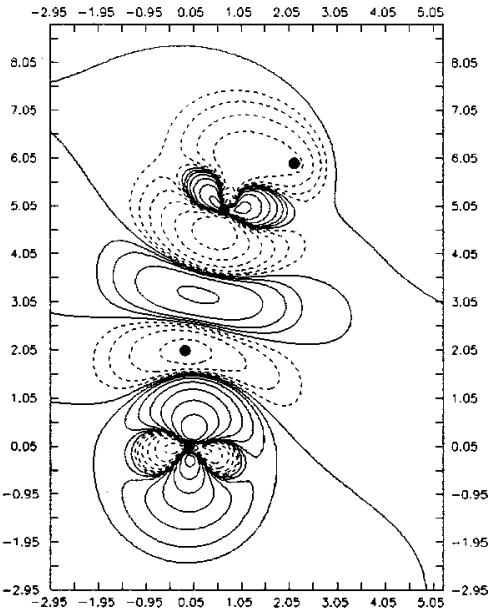

(a)

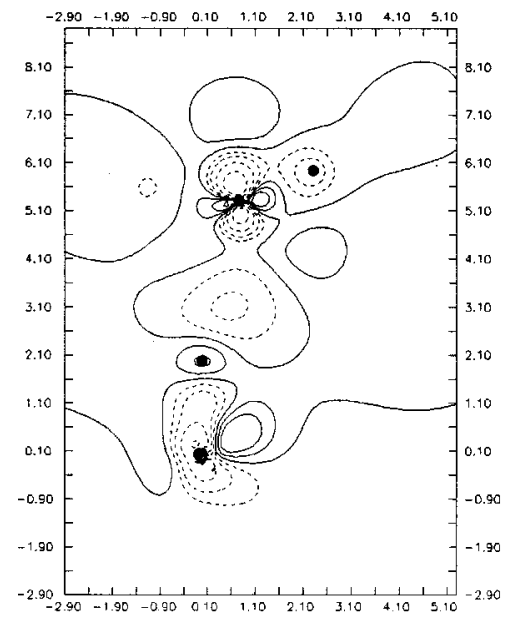

(d)

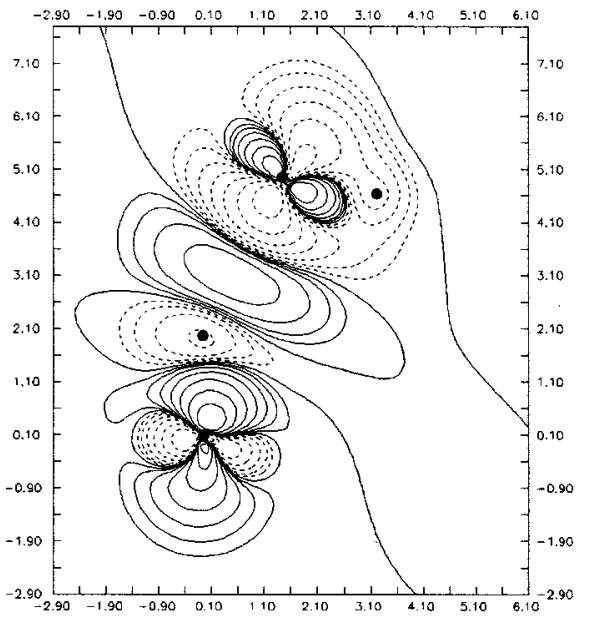

(b)

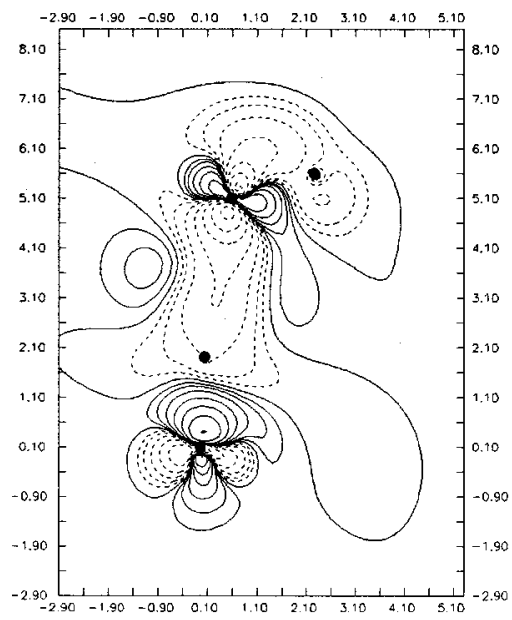

(e)

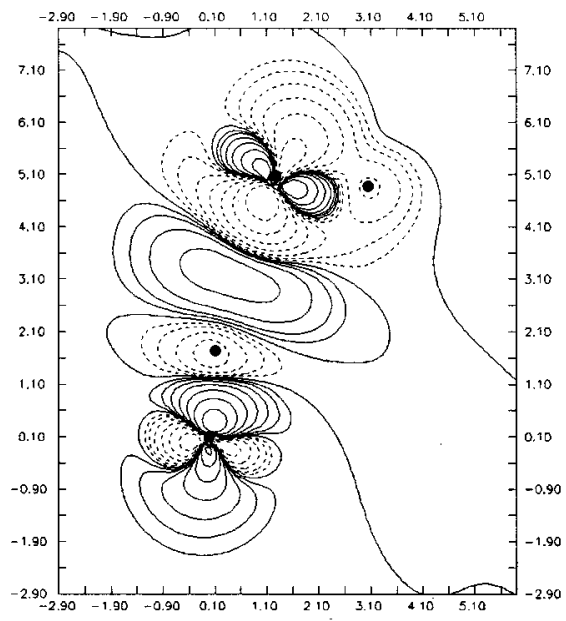

(c)

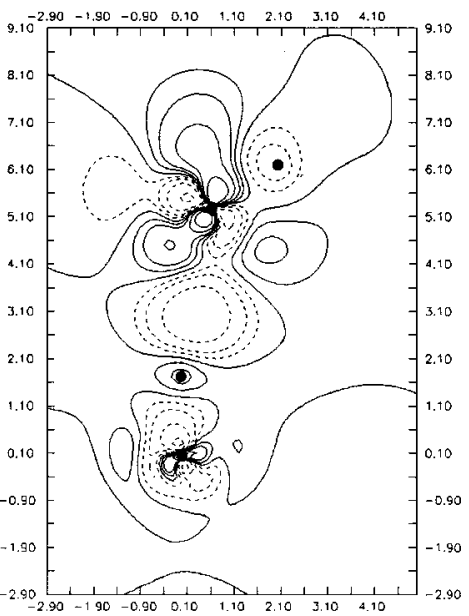

(f)

FIG. 2. SCF//SCF-CHA//SCF density difference isocontour maps: (a) 6-31G, (b) 6-31G(d), (c) 6-31G(d,p), (d) 6-31++ G(d,p), (e) 6-311 G(d,p), and (f) $6-311++\mathrm{G}(3 \mathrm{df}, 2 \mathrm{pd})$. The nuclear positions are indicated by solid dots. Solid line indicates positive values. Isodensity contours at $1 . e^{-4}, 2 . e^{-4}, 4 . e^{-4}$, $8 . e^{-4}$, etc.

density on the $\mathrm{F}$ atoms, thus avoiding a larger interaction. Therefore, after correction for the BSSE, a decrease of $\rho(r)$ is observed along the intermolecular bond zone, together with an increase of the density in the valence regions of both atoms. The situation should be the same when diffuse functions are available for the $\mathrm{H}$ and $\mathrm{F}$ atoms. However, in this case, as the intermolecular bcp falls into the van der Waals radius of both the $\mathrm{H}$ and $\mathrm{F}$ atoms, the main part of the density at the bcp is furnished by the orbitals with a larger contribution of the diffuse functions. The final effect of the BSSE correction on the electron density is the opposite of that for basis sets without diffuse functions. Thus, the charge density decreases at the $\mathrm{H}$ atom but increases slightly at the intermolecular bond region after correcting for BSSE. One must say that the density differences on the intermolecular bond region are on the order of 0.0002 a.u. and have to be seen as a consequence of the changes on the atomic basins of the $\mathrm{F}$ atoms, which have proved to be more important.

On the other hand, nuclear relaxation effects can be understood by the subsequent $\mathrm{CHA} / \mathrm{SCF} \rightarrow \mathrm{CHA} / / \mathrm{CHA}$ transi- tion. The general trend, observed for all cases but the 6-31G basis set, is the following: after BSSE correction, charge density is more concentrated in the lone pairs of the $\mathrm{F}$ atoms. Therefore the electron repulsion increases, and when nuclear relaxation is allowed, the intermolecular $r \mathrm{~F}-\mathrm{F}$ parameter increases. The distance of the bond critical point to the $\mathrm{F}$ atom of the $\mathrm{H}$ acceptor also augments and finally, both the electron density and its Laplacian at this bcp decrease to their minimum value.

When considering the whole process, SCF//SCF $\rightarrow \mathrm{CHA} / \mathrm{CHA}$, it is worth noting that the electron density at the bcp decreases in all cases, in good agreement with the weakening of the intermolecular interaction after BSSE correction, whereas the behavior of its Laplacian depends upon the precise location of this bcp. Hence, the Laplacian decreases slightly when the bcp sets closer to the F atom when correcting for BSSE. However, the opposite trend is found when the bcp is located further from the F atom after the BSSE correction. 


\section{B. DFT}

Dynamic correlation has been included through DFT with the BLYP and B3LYP exchange-correlation functionals. As commented on in Sec. IV A, the effect of BSSE on the molecular geometry is very important. All BSSEuncorrected optimizations involving basis sets without diffuse functions lead to cyclic structures (see Fig. 1), which should normally correspond to a transition state connecting two equivalent linear conformations in an internal rotation process. ${ }^{30}$ Moreover, all CHA optimized structures yield the correct, nearly linear arrangement, in good agreement with experimental ${ }^{27}$ data. Therefore, for this system, CHA deals well with BSSE-like delocalizations occurring even when rather incomplete basis sets are used, modifying the topology of the PES.

The results of a Bader analysis of the charge density are collected in Tables V and VI. Again the values of the electron density and its Laplacian at the bond critical points reveal a closed-shell interaction between the monomers. Interestingly, for uncorrected BLYP, with all the basis sets without diffuse functions (except 6-311G(d,p)), one finds a ring critical point (rcp) and two bond critical points, almost equivalent due to the symmetry, between both $\mathrm{H}-\mathrm{F}$ molecules. That is, both monomers act as $\mathrm{H}$ donor and $\mathrm{H}$ acceptor at the same time and the bonding is described by two intermolecular interactions between both hydrogen fluoride monomers. For the rest of the calculations only a bcp is located on the charge density in the intermolecular region. Using the uncorrected B3LYP functional changes this situation dramatically. Then, only the smallest basis set, 6-31G, yields a rcp, even though cyclic structures are found for the rest of basis sets without diffuse functions. In terms of the geometrical parameters obtained for both functionals, one could argue that both yielded bad descriptions of the bonding when BSSE is not taken into account. However, Bader analysis shows that a cyclic structure is not always associated with the existence of two hydrogen bonds in the BSSEuncorrected system.

In fact, up to four different connectivities are observed for these cyclic structures. When a single intermolecular bcp is located on the charge density, the intermolecular bond path links both $\mathrm{F}$ atoms. This molecular graph is also observed for the cyclic transition state structures obtained at the SCF level of theory. When the electron density exhibits a rcp there are several possible connectivities. For instance, with the 6-31G basis set using the BLYP functional, both the uncorrected and the CHA corrected electron density show a molecular graph, where the two intermolecular bcps connect each $\mathrm{F}$ atom with the $\mathrm{H}$ atom of the partner. When the basis set size is increased with polarization functions two bond paths connecting the $\mathrm{F}$ atoms are observed. Finally, the B3LYP/6-31G electron density exhibits a rather unphysical molecular graph with one bond path connecting the two $\mathrm{F}$ atoms and another one linking one of the $\mathrm{F}$ atoms with the $\mathrm{H}$ atom of the other monomer. Indeed, the electron density surface is so flat in the intermolecular region that small perturbations can induce dramatic changes on its topology and connectivity. The main conclusion is that the cyclic structure is very unstable and that the electronic relaxation caused by the BSSE correction is unable to overcome this circumstance.

To investigate the effect of the CHA correction on the charge density we refer again to the electronic relaxation process described by the transition DFT//DFT $\rightarrow$ CHA//DFT. In general, rather small differences in the values of the charge density and its Laplacian are found again and trends similar to those found above for the SCF calculations are observed for some basis sets. However, for basis sets yielding rcps, the effect of the electronic relaxation is very important. Regarding BLYP results, it can be seen that in all cases the rcp is slightly moved away from the $\mathrm{F}$ atom of the original $\mathrm{H}$-acceptor molecule, which induces a decrease of the electron density and an increase of its Laplacian. However, whereas the rank of the critical point is not modified for the BLYP/6-31G and BLYP/6-31G(d,p) basis sets after CHA correction, it is modified when adding polarization functions to $\mathrm{H}$ atoms. In such a case, the critical point is displaced along the opposite direction. Therefore, the two new bond critical points observed between both BSSE-uncorrected $\mathrm{F} \cdot \mathrm{H}$ intermolecular interactions before the CHA correction is applied disappear, so the hydrogen bonding becomes described by a unique bcp corresponding to a single hydrogen bond.

Concerning B3LYP calculations, the ring critical point is found only for the smallest basis set. Again, after electronic relaxation the topology of the charge density changes and a single bond critical point is found. In this case, both the electron density and its Laplacian decrease; the most important effect is, however, the displacement of the critical point away from the $\mathrm{F}$ atom of the $\mathrm{H}$-acceptor molecule by more than $0.05 \AA$. The final distance of $1.199 \AA$ is much closer to those obtained with larger basis sets.

Density difference maps at DFT levels of theory are collected in Fig. 3. All maps have been obtained at the uncorrected geometry for each basis set. Maps for basis sets with and without diffuse functions were very similar among them, so in order to save space, only those corresponding to the $6-31 \mathrm{G}(\mathrm{d}, \mathrm{p}), 6-31 \mathrm{G}++(\mathrm{d}, \mathrm{p})$, and $6-311 \mathrm{G}(\mathrm{d}, \mathrm{p})$ are presented. Interestingly, there are almost no differences between the maps obtained with BLYP or B3LYP functionals, despite their rather different effect on the topology of the charge density, and the effect of the electronic relaxation.

Graphical representations for the basis sets including diffuse functions are as well very similar to the SCF ones [see Figs. 3(b) and 3(e)]. The main differences are located in the vicinity of the $\mathrm{F}$ atoms, mainly those of the $\mathrm{H}$-acceptor molecule. Furthermore, the large effect of the electronic relaxation for the rest of the basis sets leading to cyclic structures is obvious from the maps [see Figs. 3(a), 3(c), 3(d), and 3(f)]. Isocontour lines increase in the vicinity of both $\mathrm{F}$ atoms. Again, CHA density is more concentrated in the lone pair regions of the F atoms and in both hydrogens, whereas BSSE overestimates the charge density in the intermolecular region and along the intramolecular $\mathrm{F} \cdots \mathrm{H}$ bond paths. This overestimation of the charge density in the intermolecular zone is more concentrated in the zone where the two new bond critical points associated with the rcp are expected to appear in the uncorrected density. Nevertheless, all maps show quali- 


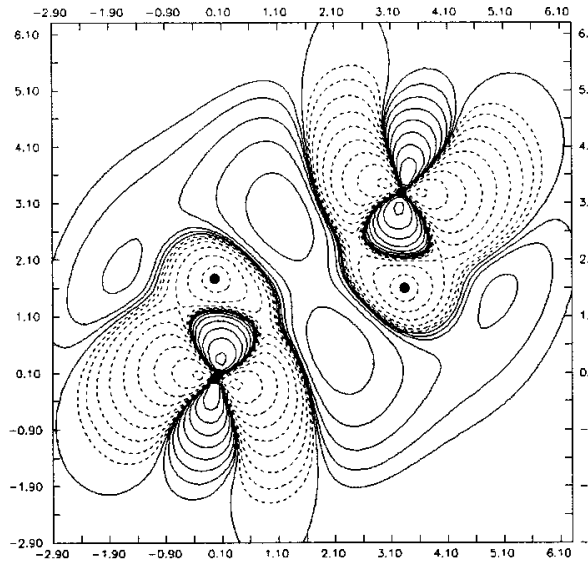

(a)

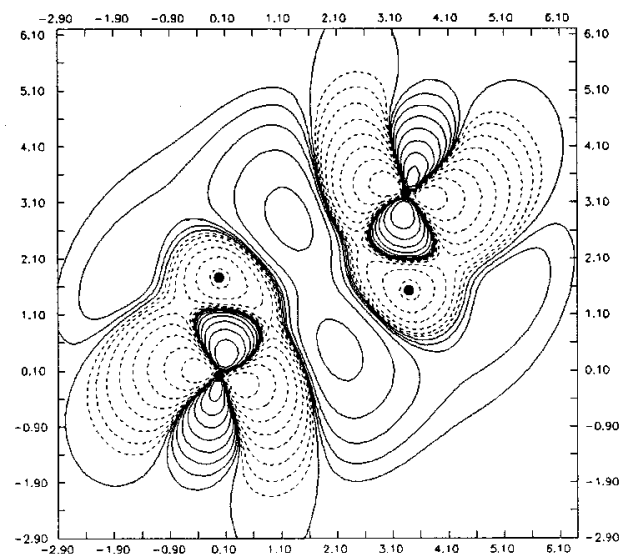

(d)

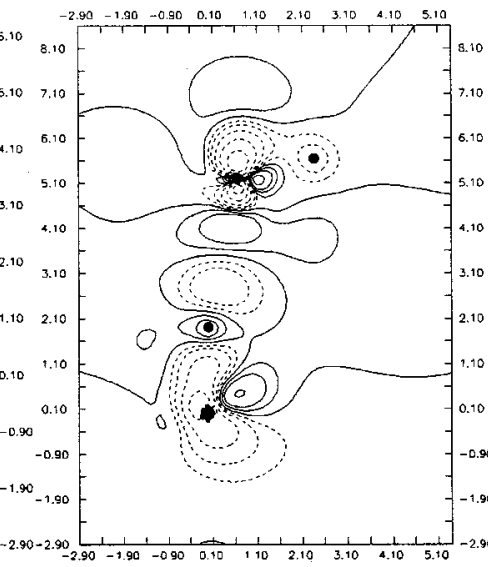

(b)

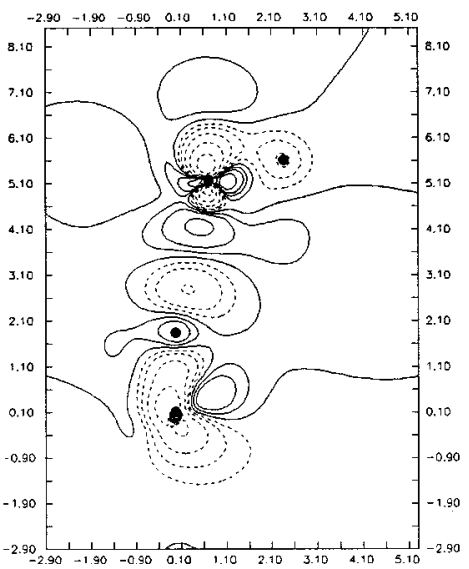

(e)

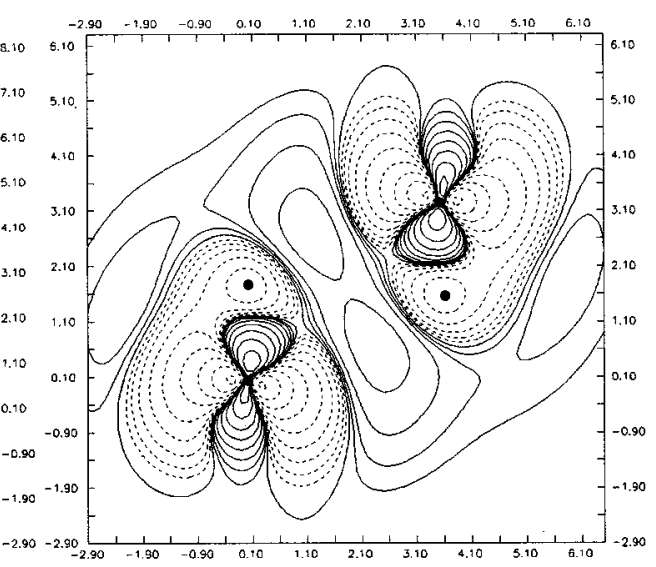

(c)

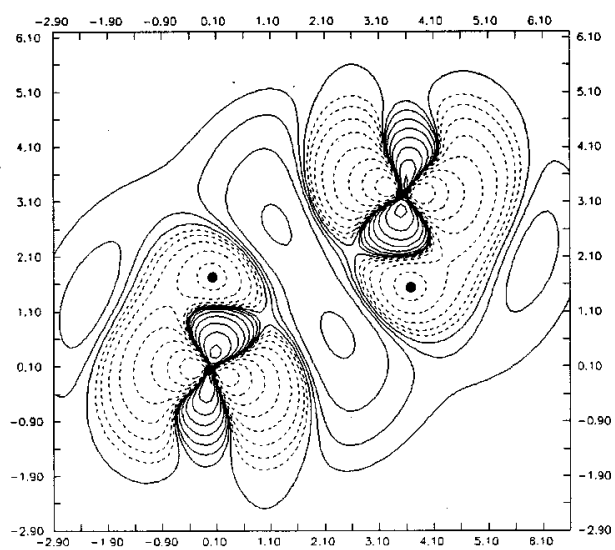

(f)

FIG. 3. DFT//DFT-CHA//DFT density difference isocontour maps: (a) BLYP/6-31G(d,p), (b) BLYP/6-31+ + G(dp), (c) BLYP/6-311G(d,p), (d) B3LYP/631G(d,p), (e) B3LYP/6-31+ + G(d,p), and (f) B3LYP/6-311G(d,p). The nuclear positions are indicated by solid dots. Solid line indicates positive values. Isodensity contours at $1 . e^{-4}, 2 . e^{-4}, 4 . e^{-4}, 8 . e^{-4}$, etc.

tatively the same trends, disregarding the existence of the rcp on the uncorrected density or whether CHA correction changes this situation.

Finally, the nuclear relaxation process is very important. In all cases the final $\mathrm{CHA} / \mathrm{CHA}$ structures have a linear arrangement and the bonding is represented by a single bond critical point in the intermolecular region. When nuclear relaxation is allowed, the intermolecular F-F distance increases by $0.2 \AA$, whereas the intermolecular F * *H distance decreases in the same way due to the changes in the angular disposition of both monomers. Of course, the main differences in terms of the location of the bcp and its density and Laplacian are observed for the basis sets that yielded cyclic structures on the uncorrected PES. The final location of the bcp is much closer to the $\mathrm{F}$ atom of the $\mathrm{H}$-acceptor molecule whereas, contrary to the cases presenting linear arrangements, the value of the charge density increases by up to $35 \%$ in some cases. There is a simple explanation for this effect. In the cyclic structure, the intermolecular $\mathrm{F} \cdots \mathrm{H}$ distances are much longer than in the linear case. The uncorrected interaction energies are of the order of $8-10 \mathrm{kcal} / \mathrm{mol}$, but this value can be seen as the sum of the contributions of two intermolecular bonds, even though the Bader analysis does not always detect their presence, e.g., at the BLYP/631G(d), BLYP/6-31 G(d,p) and BLYP/6-311 G(d,p) levels of theory. Under this assumption, the interaction energy per intermolecular bond is always larger for the CHA//CHA calculation than for the DFT/DFT in the cyclic cases; therefore its seems reasonable to expect a larger value of the density at the corresponding bcp. Changes in the Laplacian are less important in general and much more erratic; is not easy to define a tendency from the tabulated values.

The differences observed after the BSSE correction for the calculations including diffuse functions are almost negligible, showing again the asymptotic approach between the uncorrected and the CHA results when the quality of the basis set is improved.

\section{CONCLUSIONS}

$A b$ initio electron densities corrected for BSSE have been obtained using the CHA method at both the SCF and DFT levels of theory using several basis sets. The charge density and its Laplacian at the critical points located on the electron density, as defined in the AIM theory, have been used to compare quantitatively uncorrected densities and their CHA counterparts at the conventional optimized geometry and after nuclear relaxation.

The effect of BSSE on the electron density has proved to be more relevant at the DFT level than at the SCF level of 
theory, and to be strongly dependent on the inclusion of diffuse functions in the basis set. B3LYP/6-31G, BLYP/631G, BLYP/6-31G(d), and BLYP/6-31 G(d,p) calculated electron densities yield a rcp and two bcps in the intermolecular bond region, thus revealing the presence of two intermolecular bonds. The electronic relaxation due to the CHA correction changes this situation only in B3LYP/6$31 \mathrm{G}$ and BLYP/6-31G(d,p) cases. According to the present results, the B3LYP functional behaves much better than BLYP in terms of topology of the charge density when small basis sets are used. Geometry optimizations lead to similar cyclic structures and BSSE values for either the BLYP or B3LYP functionals; therefore one must conclude that, for the hydrogen fluoride dimer, the topology of the electron density is much more sensitive to BSSE than the PES or the interaction energy. Indeed, for the cyclic structures the electron density surface is so flat in the intermolecular region and the different rcps are so close to each other, that small perturbations can easily lead to changes of its topology.

When the uncorrected PES is qualitatively well described without the BSSE correction, both electronic and nuclear relaxation lead to deviations on the order of $10 \%$ in the intermolecular critical point location, its electron density, and Laplacian. The convergence of uncorrected and CHA results for the largest basis set shows again the validity of this BSSE-correction scheme.

Density difference maps show that the leading process occurring after correcting for BSSE is the transfer of electron density from the intramolecular $\mathrm{F}-\mathrm{H}$ bonds to the $\mathrm{F}$ lone electron pairs, rather than the depletion of charge density in the intermolecular region, which in fact has proved to strongly depend upon the inclusion of diffuse functions. This situation had also been pointed out by Gatti et al. ${ }^{17}$ for the water dimer using the SCF-MI method and a large basis set.

Finally, nuclear relaxation has proved to be essential in order to obtain a reliable BSSE-corrected description of the hydrogen fluoride dimer. The effects of the BSSE corrections on the electronic densities of the complex studied are completely consistent with the results obtained previously for the corrected CHA interaction energies.

\section{ACKNOWLEDGMENTS}

This work has been funded through Spanish DGES Project No. PB98-0457-C02-01. One of us (P.S.) acknowledges the financial support by CIRIT Grant No. 1998FI-
00564. The authors are grateful to Dr. B. Paizs for providing a copy of the CHA programs, and to Dr. I. Mayer for useful discussions.

${ }^{1}$ H. B. Jansen and P. Ros, Chem. Phys. Lett. 3, 140 (1969).

${ }^{2}$ F. B. Van Duijneveldt, J. G. C. M. Van Duijneveldt-van de Rijt, and J. H. Van Lenthe, Chem. Rev. 94, 1873 (1994) and references therein.

${ }^{3}$ S. F. Boys and F. Bernardi, Mol. Phys. 19, 553 (1970).

${ }^{4}$ S. Simon, M. Duran, and J. J. Dannenberg, J. Chem. Phys. 105, 11024 (1996).

${ }^{5}$ I. Mayer and Á. Vibók, Chem. Phys. Lett. 136, 115 (1987).

${ }^{6}$ G. Halász, Á. Vibók, and S. Suhai, Int. J. Quantum Chem. 68, 151 (1998).

${ }^{7}$ I. Mayer and Á. Vibók, Int. J. Quantum Chem. 15, 139 (1991).

${ }^{8}$ G. Hálasz, Á. Vibók, P. Valiron, and I. Mayer, J. Phys. Chem. A 100, 6332 (1996)

${ }^{9}$ B. Paizs and S. Suhai, J. Comput. Chem. 18, 694 (1997).

${ }^{10}$ I. Mayer and P. Valiron, J. Chem. Phys. 109, 3360 (1998).

${ }^{11}$ I. Mayer and Á. Vibók, Mol. Phys. 92, 503 (1997).

${ }^{12}$ I. Mayer, Á. Vibók, and P. Valiron, Chem. Phys. Lett. 224, 166 (1994).

${ }^{13}$ P. Salvador, B. Paizs, M. Duran, and S. Suhai, J. Comput. Chem. (submitted).

${ }^{14}$ B. Paizs and S. Suhai, J. Comput. Chem. 19, 575 (1998).

${ }^{15}$ R. F. W. Bader, Atoms in Molecules: A Quantum theory (Clarendon, Oxford, 1990).

${ }^{16}$ M. Solà, J. Mestres, R. Carbó, and M. Duran, J. Chem. Phys. 104, 636 (1996); K. B. Wiberg, C. M. Hadad, T. J. LePage, C. M. Breneman, and M. J. Frisch, J. Phys. Chem. 96, 671 (1992).

${ }^{17}$ C. Gatti and A. Famulari, Interaction Energies and Densities. A Quantum Theory of Atoms in Molecules Insight on the Effect of Basis Set Superposition Error Removal, Understanding Chemical Reactivity: Electron, Spin and Momentum Densities and Chemical Reactivity, Kluwer book series, Vol. 2, edited by P. G. Mezey and B. Robertson (Kluwer Dordrecht, The Netherlands, 1998).

${ }^{18}$ M. Raimondi, A. Famulari, and E. Gianinetti, Int. J. Quantum Chem. 74, 259 (1999).

${ }^{19}$ E. Gianinetti, M. Raimondi, and E. Tornaghi, Int. J. Quantum Chem. 60, 157 (1996).

${ }^{20}$ I. Mayer, Int. J. Quantum Chem. 70, 41 (1998).

${ }^{21}$ B. Paizs and I. Mayer, Chem. Phys. Lett. 220, 97 (1994).

${ }^{22}$ M. J. Frisch et al., GAUSSIAN 94, Gaussian, Inc., Pittsburgh PA (1995).

${ }^{23}$ M. J. Frisch, GAussian 92, Gaussian, Inc., Pittsburgh PA (1992).

${ }^{24}$ F. W. Biegler-König, R. F. W. Bader, and T. H. Tang, J. Comput. Chem. 3, 317 (1982).

${ }^{25}$ In the sense that molecular properties found with the BSSE-corrected procedures are close to the ones obtained with much larger basis sets.

${ }^{26}$ P. Salvador and M. Duran, J. Chem. Phys. 111, 4460 (1999).

${ }^{27}$ B. J. Howard, T. R. Dyke, and W. Klemperer, J. Chem. Phys. 81, 1315 (1995).

${ }^{28}$ P. Salvador, S. Simon, M. Duran, and J. J. Dannenberg, J. Chem. Phys. (submitted).

${ }^{29}$ J. E. Del Bene, W. B. Pearson, and K. Szczepaniak, J. Phys. Chem. 99, 10705 (1995) and references therein.

${ }^{30}$ For instance, at the SCF/6-31G(d) level of theory, a cyclic structure is located on the PES with an imaginary frequency of $150 i \mathrm{~cm}^{-1}$. Similarly, with the CHA/DFT method using the same basis set, another cyclic structure is located on the PES and characterized as a transition state with a similar imaginary frequency. 